OPEN ACCESS

Edited by: Jihong Bai, Fred Hutchinson Cancer Research Center, USA

Reviewed by: Thomas F. J. Martin, University of Wisconsin-Madison,

University of Trento, Italy

Sung Hyun Kim,

Kyung Hee University School of Medicine, South Korea

*Correspondence:

Robert H. Edwards robert.edwards@ucsf.edu

Received: 07 October 2016 Accepted: 10 February 2017 Published: 23 February 2017

Citation:

Maas JW Jr., Yang J and Edwards RH (2017) Endogenous Leucine-Rich Repeat Kinase 2 Slows Synaptic Vesicle Recycling in Striatal

Neurons.

Front. Synaptic Neurosci. 9:5. doi: 10.3389/fnsyn.2017.00005

\section{Endogenous Leucine-Rich Repeat Kinase 2 Slows Synaptic Vesicle Recycling in Striatal Neurons}

\author{
James W. Maas Jr., Jing Yang and Robert H. Edwards * \\ Departments of Neurology and Physiology, Weill Institute for Neurosciences, and Kavli Institute for Fundamental \\ Neuroscience, UCSF School of Medicine, San Francisco, CA, USA
}

Dominant mutations in leucine-rich repeat kinase 2 (LRRK2) produce the most common inherited form of Parkinson's disease (PD) but the function of LRRK2 remains poorly understood. The presynaptic role of multiple genes linked to PD including $\alpha$-synuclein ( $\alpha$-syn) has suggested that LRRK2 may also influence neurotransmitter release, a possibility supported by recent work. However, the use of disease-associated mutants that cause toxicity complicates the analysis. To determine whether LRRK2 normally influences the synaptic vesicle, we have now used a combination of imaging and electrophysiology to study LRRK2 knockout (KO) mice. Surprisingly, we find that in hippocampal (generally excitatory) neurons, the loss of LRRK2 does not affect synaptic vesicle exocytosis, endocytosis or the mobility of $\alpha$-syn. Double KO (DKO) mice lacking LRRK1 as well as LRRK2 also show no defect in transmitter release by hippocampal neurons. However, in striatal neurons, which express LRRK2 at higher levels, the loss of LRRK2 leads to modest acceleration of synaptic vesicle endocytosis. Thus, endogenous LRRK2 normally slows synaptic vesicle recycling at striatal terminals.

Keywords: LRRK2, endocytosis, synaptic vesicle, Parkinson's disease, striatum

\section{INTRODUCTION}

Although Parkinson's disease (PD) is defined by the loss of dopamine neurons in the substantia nigra, accumulating evidence suggests that pathology originates at the nerve terminal (Nakata et al., 2012; Janezic et al., 2013). The protein $\alpha$-synuclein ( $\alpha$-syn) that causes familial and idiopathic forms of PD accumulates in dystrophic axonal processes as well as somatic Lewy bodies (Polymeropoulos et al., 1997; Spillantini et al., 1997, 1998; Singleton et al., 2003). Indeed, $\alpha$-syn normally localizes to the nerve terminal (George et al., 1995; Iwai et al., 1995), inhibits neurotransmitter release when over-expressed (Greten-Harrison et al., 2010; Nemani et al., 2010; Scott et al., 2010; Anwar et al., 2011; Janezic et al., 2013) and may promote formation of the SNARE complex that mediates synaptic vesicle exocytosis (Burré et al., 2010). PD-associated mutations in the endocytic proteins synaptojanin and auxilin further support a presynaptic locus for the disorder (Edvardson et al., 2012; Quadri et al., 2013).

In contrast to the rare mutations in $\alpha$-syn, synaptojanin and auxilin, dominant mutations in leucine-rich repeat kinase 2 (LRRK2) produce the most common form of inherited PD and up to $10 \%$ of sporadic disease (Healy et al., 2008). LRRK2 is a multi-domain protein with roles reported in the endolysosomal pathway (Dodson et al., 2012; Gómez-Suaga et al., 2014), autophagy (Orenstein et al., 2013), translation (Martin et al., 2014) and transmitter release. Since the pathology resulting from LRRK2 mutations usually involves deposition of $\alpha$-syn (Giasson et al., 2006), it is possible that LRRK2, like $\alpha$-syn, modulates neurotransmitter release, and that mutant LRRK2 causes disease through a presynaptic mechanism, possibly involving synuclein. 
LRRK2 localizes to the nerve terminal, but in contrast to $\alpha$-syn, is not exclusively presynaptic (Biskup et al., 2006). Nonetheless, LRRK2 has been reported to interact with multiple presynaptic proteins, including NEM-sensitive factor (NSF) and the t-SNARE syntaxin-1, and may bind to purified synaptic vesicles, whereas loss of LRRK2 alters the density and size of synaptic vesicles (Piccoli et al., 2011, 2014; Cirnaru et al., 2014; Arranz et al., 2015; Belluzzi et al., 2016). LRRK2 has also been reported to influence synaptic physiology. Mice overexpressing wild type (WT) or mutant LRRK2 show opposing effects on dopamine release in the striatum (Li et al., 2009, 2010), suggesting a presynaptic site of action. Modest, transient changes in synaptic activity have been reported in mice expressing mutant LRRK2 and LRRK2 knockout (KO) mice, but these may reflect the modification of dendritic spines (Parisiadou et al., 2014; Matikainen-Ankney et al., 2016). Moreover, another study was unable to demonstrate any purely presynaptic abnormalities in LRRK2 KO mice or mice over-expressing WT LRRK2 (Beccano-Kelly et al., 2015). In cultured neurons, RNAi-mediated silencing of LRRK2 has been reported to promote synaptic vesicle exocytosis (Piccoli et al., 2011), but other work has suggested changes in endocytosis due to loss of LRRK2 or expression of mutant LRRK2 (Shin et al., 2008; Matta et al., 2012; Arranz et al., 2015; Belluzzi et al., 2016).

Since PD-associated mutants can produce toxicity that complicates the analysis of presynaptic function, we have now used KO mice and a combination of optical and electrophysiological techniques to elucidate the role of LRRK2 in the synaptic vesicle cycle. We demonstrate that loss of LRRK2 does not alter basal synaptic transmission, and does not alter the synaptic vesicle cycle in cultured glutamatergic hippocampal neurons. Further, the loss of LRRK2 is not compensated for by residual LRRK1. However, in striatal neurons, which express endogenous LRRK2 at high levels, loss of LRRK2 leads to a modest acceleration of endocytosis. Thus, LRRK2 normally inhibits synaptic vesicle recycling in striatal neurons.

\section{MATERIALS AND METHODS}

\section{Animals}

This study was carried out in strict accordance with the recommendations in the Guide for the Care and Use of Laboratory Animals of the National Institutes of Health. The protocol was approved by the Institutional Animal Care and Use Committee of the University of California, San Francisco (protocol number: AN109510). All efforts were made to minimize suffering. LRRK1 KO mice were generated by the Michael J. Fox Foundation and both LRRK1 and 2 KO (Lin et al., 2009) mice obtained from Jackson Labs. Due to reduced survival on the $\mathrm{C} 57 \mathrm{Bl} / 6$ background, heterozygous LRRK1 mice on this background were crossed with FVB mice to produce LRRK1 KO mice. LRRK1 KO and WT littermate controls were generated from heterozygous LRRK1 parents. We used LRRK1 heterozygous/LRRK2 KO breeding pairs to produce LRRK1 KO/LRRK2 KO mice and LRRK1 WT/LRRK2 KO littermates as control. Animals were genotyped by PCR.

\section{Neuronal Culture and Transfection}

To produce mouse hippocampal cultures, postnatal day 0 hippocampus was digested in papain $(20 \mathrm{U} / \mathrm{ml}$, Worthington), washed, triturated and plated on glass coverslips pre-coated with poly-L-lysine at a density of 700 cells $/ \mathrm{mm}^{2}$. Neurons were plated in minimal essential media containing 5\% FBS, B27 supplement (Gibco), $21 \mathrm{~mm}$ glucose, $2 \mathrm{mM}$ glutamax and Mito+ serum extender (VWR). Twenty-four hours after plating, $75 \%$ of the medium was removed and replaced with medium containing Neurobasal, B27 supplement, and $2 \mathrm{mM}$ glutamax. After 7 days in culture, one third of the medium was exchanged, and $4 \mu \mathrm{M}$ cytosine arabinoside added to prevent glial proliferation. Neurons were transfected using calcium phosphate at 6-7 days in vitro (DIV). To produce mixed cortical/striatal neuron cultures, the medial ganglionic eminence and cortex were isolated from embryonic day 16 mouse embryos, digested in trypsin (2.5\%, Gibco) and DNase I (10 mg/ml, Roche), washed and triturated. Striatal neurons were transfected by electroporation (Amaxa), mixed with untransfected cortical neurons (3:2 striatal:cortical) and plated on glass coverslips pre-coated with poly-L-lysine at a density of 1100 cells $/ \mathrm{mm}^{2}$ in Neurobasal medium containing B27 supplement and $2 \mathrm{mM}$ glutamax. Medium was exchanged and cytosine arabinoside added as described for hippocampal cultures.

\section{Imaging}

Hippocampal neurons were transfected with the $\mathrm{pH}$-sensitive GFP variant superecliptic pHluorin, targeted to the lumenal domain of synaptic vesicles by insertion into the large lumenal loop of vesicular glutamate transporter 1 (VGLUT1-pHluorin; Voglmaier et al., 2006). Striatal neurons were transfected with the vesicular GABA transporter (VGAT) tagged with pHluorin at the lumenal/extracellular C-terminus (VGATpHluorin; Santos et al., 2013). Neurons were imaged between DIV 14-17, as previously described (Nemani et al., 2010). Briefly, coverslips containing neurons were placed in an RC-21BRFS imaging chamber containing bipolar stimulating electrodes (Warner Instruments) in closed configuration. Neurons were continuously perfused $(1 \mathrm{ml} / \mathrm{min})$ at room temperature with Tyrode's solution (in mM: $119 \mathrm{NaCl}, 2.5 \mathrm{KCl}, 2 \mathrm{CaCl}_{2}$, $2 \mathrm{MgCl}_{2}, 30$ glucose and 25 HEPES, $\mathrm{pH}$ 7.4) containing glutamate receptor antagonists 6-cyano-7 nitroquinoxaline-2, 3-dione (CNQX; $10 \mu \mathrm{M}$ ) and D, L-2-amino-5-phosphonovaleric acid (APV; $50 \mu \mathrm{M})$ and stimulated using trains of $1 \mathrm{~ms}$ current pulses to yield fields of $5-10 \mathrm{~V} / \mathrm{cm}$. We usually delivered a train of 600 stimuli at $10 \mathrm{~Hz}$ for $60 \mathrm{~s}$, but in some experiments we delivered shorter trains at alternate frequencies, as indicated in the text. $\mathrm{NH}_{4} \mathrm{Cl}$ buffer (in mM: $69 \mathrm{NaCl}$, $2.5 \mathrm{KCl}, 2 \mathrm{MgCl}_{2}, 2 \mathrm{CaCl}_{2}, 50 \mathrm{NH}_{4} \mathrm{Cl}, 30$ glucose, 25 HEPES, $\mathrm{pH}$ 7.4) was used to reveal total pHluorin fluorescence. Images were collected under epifluorescence illumination using 
a Nikon TE300 inverted microscope and xenon lamp with ET470/40 nm excitation and ET525/50 nm emission bandpass filters, a $63 \times 1.2$ N.A. water objective, and a EM-CCD camera (Photometrics QuantEM:512SC) controlled by Metamorph software.

Initial rate of fluorescence increase was calculated by determining the slope of a line through the data point immediately prior to stimulation and the first two points during stimulation. To specifically assess exocytosis, we stimulated neurons at 10 or $20 \mathrm{~Hz}$ for $150 \mathrm{~s}$ in the presence of bafilomycin $(0.6 \mu \mathrm{M}$, diluted from $1000 \times$ stock solutions in DMSO). Initial exocytic rate in bafilomycin was calculated by determining the slope of a line through the data points in the first $10 \mathrm{~s}$ after start of stimulation, when the increase in fluorescence is linear.

Endocytosis rate was calculated by normalizing curves to the peak response at the end of stimulation and fitting a single exponential decay curve to determine the decay constant, $\tau$.

For destaining experiments, neurons were loaded by stimulation at $10 \mathrm{~Hz}$ for $2 \mathrm{~min}$ in $15 \mu \mathrm{M}$ FM4-64 (ThermoFisher) in Tyrode's solution, allowed to recover for $2 \mathrm{~min}$, washed briefly with Tyrode's solution containing $1 \mathrm{mM}$ Advasep-7 (Biotium) followed by a longer wash with Tyrode's solution, and images were collected as above using ET 470/40 nm excitation and 650 longpass emission filters. Curves were normalized to the initial peak fluorescence and fitted with a single exponential decay curve to determine the time constant $\tau$, reflecting the loss of dye and by inference, the rate of exocytosis.

\section{Fluorescence Recovery after Photobleaching}

Neurons were imaged between DIV 14-17 in Tyrode's solution with $10 \mu \mathrm{M}$ CNQX and $50 \mu \mathrm{M}$ APV at $37^{\circ} \mathrm{C}$ using a Zeiss LSM 510 confocal microscope with $63 \times$ oil immersion objective (NA 1.4) and $488 \mathrm{~nm}$ argon laser line. The pinhole was set to $2.5 \mu \mathrm{m}$ to collect light from the entire bouton and images were collected every $400 \mathrm{~ms}$ at $1.5 \%$ transmission to reduce bleaching. For photobleaching, 40 consecutive scans were performed over $1 \mathrm{~s}$ at $100 \%$ transmission. The curves for fluorescence recovery after photobleaching (FRAP) were fit to a single exponential to calculate the time constant for recovery, $\tau$.

\section{Electrophysiology}

Acute hippocampal slices were prepared from 3 week old mice. Three-hundred and fifty micrometer hippocampal slices were cut with a vibratome in aerated slicing solution (in $\mathrm{mM}, 228$ sucrose, $2.5 \mathrm{KCl}, 1 \mathrm{NaH}_{2} \mathrm{PO}_{4}, 7 \mathrm{MgSO}_{4}, 0.5 \mathrm{CaCl}_{2}$, $26 \mathrm{NaHCO}_{3}$ and 11 dextrose) at $4^{\circ} \mathrm{C}$, transferred to artificial cerebrospinal fluid (aCSF; in mM, $119 \mathrm{NaCl}, 2.5 \mathrm{KCl}, 1.3 \mathrm{MgSO}_{4}$, $2.5 \mathrm{CaCl}_{2}, 26 \mathrm{NaHCO}_{3}, 1 \mathrm{NaH}_{2} \mathrm{PO}_{4}$ and 11 dextrose adjusted to $315 \mathrm{mosmol} / \mathrm{L}$ and $\mathrm{pH} 7.4$ ) at $35^{\circ} \mathrm{C}$ and allowed to recover for a minimum of $1 \mathrm{~h}$. Slices were visualized with an upright infrared differential interference contrast microscope and perfused with standard aCSF at room temperature. Stimulation (100 $\mu \mathrm{s})$ pulse was delivered with a bipolar metal electrode (MX21AEW;
FHC). Synaptic strength was quantified as the initial slope of field potentials recorded with aCSF-filled microelectrodes $(1-2 \mathrm{M} \Omega$ ).

\section{Statistical Analysis}

Data are presented as mean \pm SEM. For experiments involving multiple measurements among genotypes, we utilized 2-way analysis of variance (ANOVA), followed by post hoc Bonferonni tests. For experiments involving a single comparison between genotypes, we used two-tailed, unpaired $t$-tests. All statistics were calculated using Graphpad Prism.

\section{RESULTS}

\section{LRRK2 and Basal Synaptic Transmission}

To determine whether the loss of LRRK2 affects synaptic transmission, we used field recordings in hippocampal slices. We first examined the input-output curve, normalizing the slope of the field potential to the strength of the fiber volley. Importantly, the loss of LRRK2 does not influence the evoked synaptic response (Figure 1A). We then assessed release probability using the paired-pulse ratio (PPR), which shows no effect of the LRRK2 $\mathrm{KO}$ except at the $20 \mathrm{~ms}$ interstimulus interval, suggesting little or no effect on neurotransmitter release (Figure 1B).

\section{Loss of LRRK2 Does Not Alter the Synaptic Vesicle Cycle}

To characterize the role of LRRK2 in synaptic vesicle cycling, we first used the styryl dye FM4-64. Like FM1-43, the red-shifted FM4-64 can be loaded into recycling synaptic vesicles by stimulation, and the subsequent stimulationdependent destaining used to monitor synaptic vesicle exocytosis (Fernández-Alfonso and Ryan, 2004). Surprisingly, hippocampal neurons from WT and LRRK2 KO mice show no difference in either the rate or extent of destaining (Figure 2A), suggesting no role for LRRK2 in either synaptic vesicle exocytosis or the size of the recycling pool.

To monitor synaptic vesicle cycling with a more sensitive reporter that also has the potential to assess endocytosis, we used the $\mathrm{pH}$-sensitive GFP variant superecliptic pHluorin, targeted to the lumenal domain of synaptic vesicles by insertion into a lumenal loop of vesicular glutamate transporter 1 (VGLUT1pHluorin; Voglmaier et al., 2006). Quenched at the low $\mathrm{pH}$ of synaptic vesicles, the fluorescence increases with exocytosis and declines with the reacidification that follows endocytosis (Miesenböck et al., 1998). We transfected this reporter into hippocampal neurons from WT and LRRK2 KO mice, and imaged the change in fluorescence in response to stimulation, normalizing to the total pool of VGLUT1-pHluorin revealed by alkalinization in $\mathrm{NH}_{4} \mathrm{Cl}\left(\Delta F_{\mathrm{NH}_{4} \mathrm{Cl}}\right)$. We again observed no difference between WT and LRRK2 KO neurons in the response to stimulation, including the initial rate of response or subsequent endocytosis (Figure 2B), with values very similar to those previously reported (Voglmaier et al., 2006; Nemani et al., 2010; Armbruster et al., 2013). However, the initial rate of fluorescence increase reflects the net contribution of both 


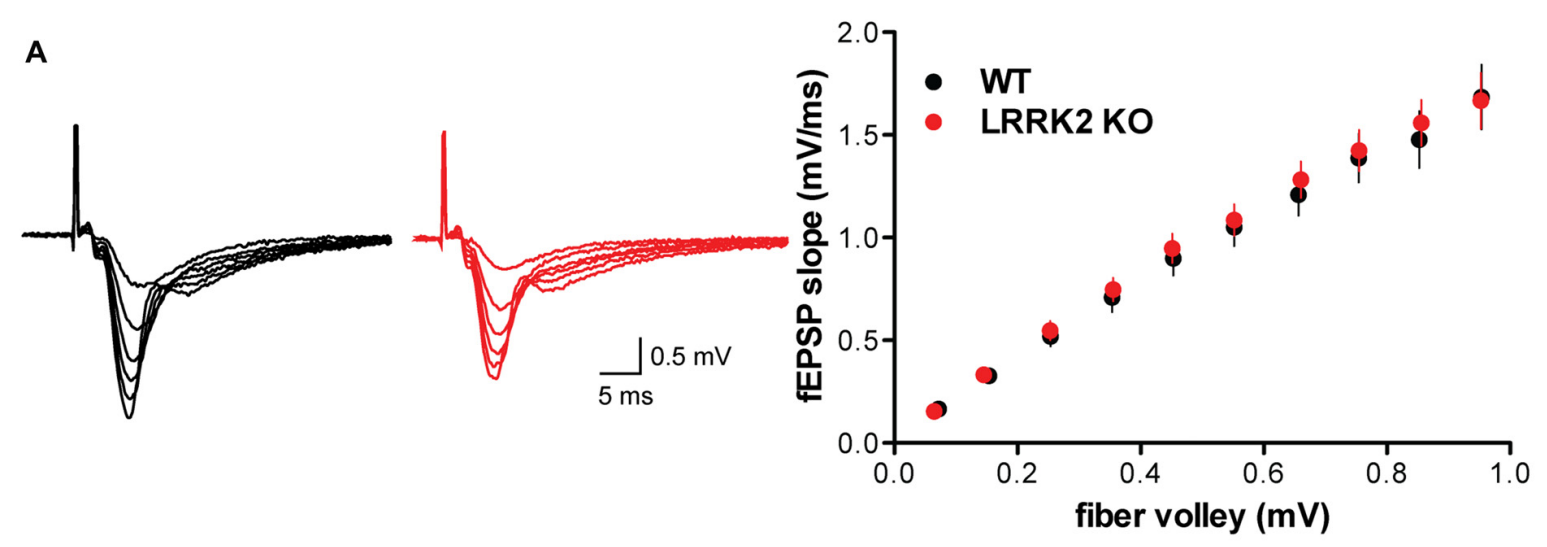

B

$|S|=20 \mathrm{~ms}$

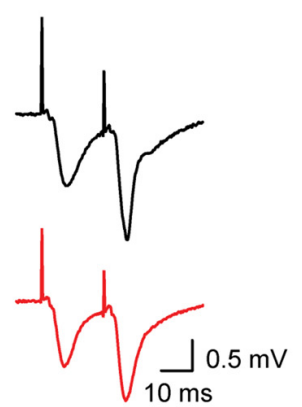

$|\mathrm{S}|=100 \mathrm{~ms}$

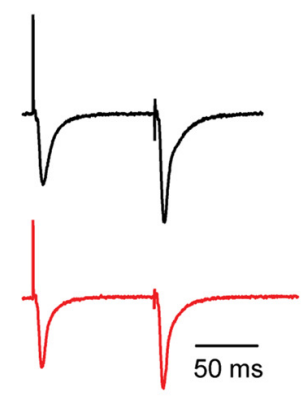

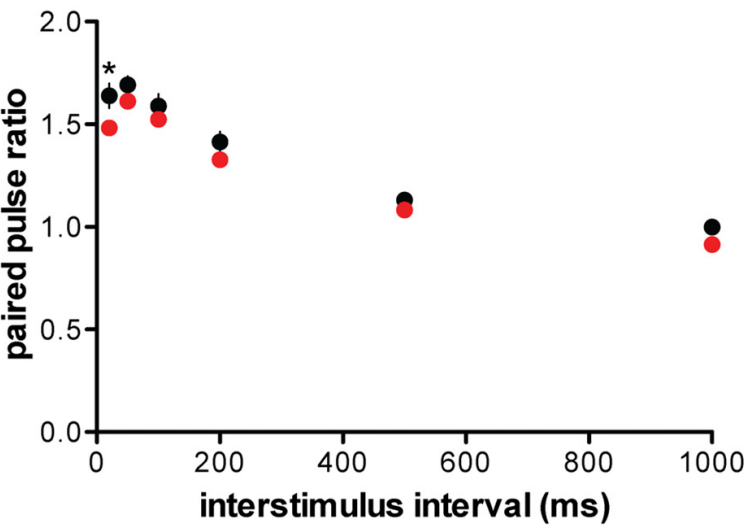

FIGURE 1 | Loss of leucine-rich repeat kinase 2 (LRRK2) does not alter basal synaptic transmission. (A) Representative traces (left) and input-output curve (right) of fEPSP slope normalized to fiber volley in hippocampal slices from wild type (WT; black) and LRRK2 knock-out (KO; red) mice $(F(1,306)=0.66, p=0.42$, $n=15-17$ slices from three animals per genotype). (B) Left, representative traces of field potentials from WT (black) and LRRK2 KO (red) slices stimulated at interstimulus intervals (ISI) of 20 and 100 ms. Right, paired pulse ratio of fEPSP in WT and LRRK2 KO hippocampal slices differs only at the 20 ms ISI $\left(F_{(1,186)}=17.53, p<0.0001,{ }^{*} p<0.05\right.$ by post-test, $n=16-17$ slices from three animals per genotype). The data represent mean \pm SEM.

exocytosis and endocytosis. To more specifically assess the impact of LRRK2 on synaptic vesicle exocytosis, we repeated the experiments in the presence of the proton pump inhibitor bafilomycin to prevent the quenching of VGLUT1-pHluorin fluorescence by reacidification of internalized vesicles, so that the response to stimulation reflects only exocytosis (Figure 2C). In the presence of bafilomycin, there was no difference between WT and LRRK2 KO neurons in the rate or extent of exocytosis, which reflects the total pool of recycling vesicles, consistent with the lack of difference in initial rates observed without bafilomycin (Figures 2B,C).

\section{LRRK2 Does Not Regulate the Mobility of $\alpha$-Synuclein}

LRRK2 may contribute to a presynaptic form of degeneration independent of synaptic vesicle cycling. Since association with membranes can influence the misfolding and aggregation of $\alpha$-syn (Davidson et al., 1998; Lee et al., 2002), we used FRAP to assess a role for LRRK2 in its mobility, which presumably reflects membrane association. In previous work, we showed that after photobleaching a single presynaptic bouton, $\alpha$-syn-GFP fluorescence recovers much more rapidly than the fluorescence of integral membrane proteins but more slowly than that of soluble GFP (Figures 3A,B), suggesting that $\alpha$-syn binds specifically but weakly to presynaptic structures (Fortin et al., 2004; Unni et al., 2010). Consistent with this interpretation, the A30P mutation disrupts presynaptic accumulation of $\alpha$-syn and accelerates recovery from photobleaching to the rate observed for soluble GFP (Fortin et al., 2004, 2005). We now find that the rate and extent of recovery for $\alpha$-syn-GFP shows no difference between hippocampal neurons from WT and LRRK2 KO mice (Figures 3A,B). LRRK2 thus does not appear to influence the mobility of $\alpha$-syn at the presynaptic terminal, and by inference, the association of $\alpha$-syn with presynaptic membranes.

\section{Loss of LRRK1 Does Not Alter Synaptic Vesicle Release}

Since loss of the single LRRK protein in Drosophila impairs synaptic vesicle endocytosis (Matta et al., 2012), we considered 


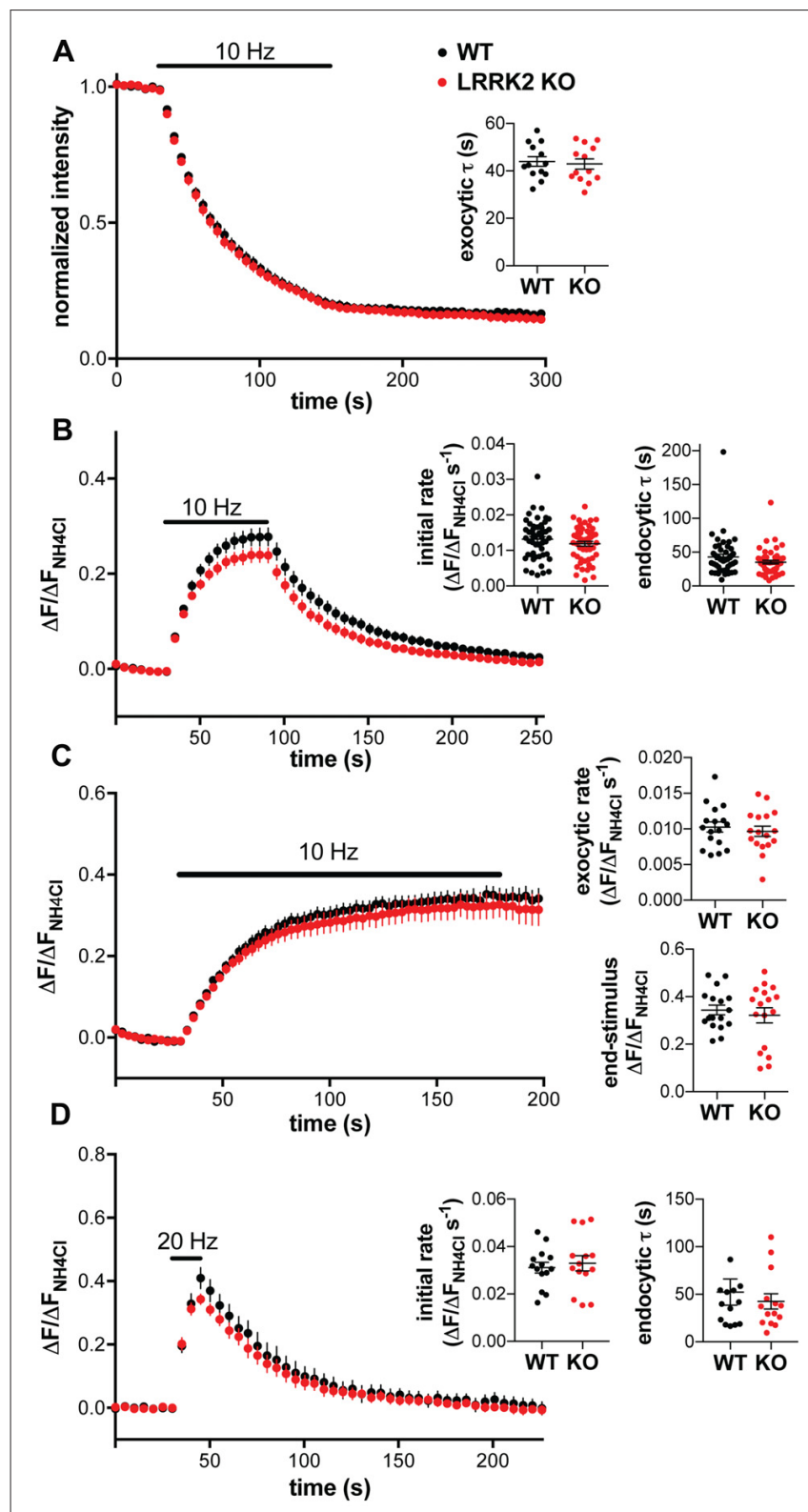

FIGURE 2 | Loss of LRRK2 does not alter synaptic vesicle exocytosis or endocytosis in hippocampal neurons. (A) Hippocampal neurons prepared from WT and LRRK2 KO mice were loaded with FM4-64 and destained by stimulation at $10 \mathrm{~Hz}$ for $120 \mathrm{~s}$. Inset shows the time constant for destaining ( $p=0.72, n=13$ coverslips per genotype). (B) Response of VGLUT1-pHluorin to $10 \mathrm{~Hz}$ stimulation for $60 \mathrm{~s}$ in transfected WT or LRRK2 KO hippocampal neurons, normalized to total VGLUT1-pHluorin revealed by alkalinization with $50 \mathrm{mM} \mathrm{NH}_{4} \mathrm{Cl}$. Insets show the initial rate of fluorescence increase $(p=0.24)$ and the time constant for endocytosis $(p=0.11) \cdot n=50-52$ coverslips per genotype. (C) Response of VGLUT1-pHluorin to $10 \mathrm{~Hz}$ stimulation for $150 \mathrm{~s}$ in the presence of bafilomycin in transfected WT or LRRK2 KO hippocampal neurons. Insets show the initial exocytic rate $(p=0.53)$ and the fluorescence at the end of stimulation ( $p=0.56) . n=17$ coverslips per genotype. (D) Response of VGLUT1-pHluorin to $20 \mathrm{~Hz}$ stimulation for $15 \mathrm{~s}$ in transfected WT or LRRK2 KO hippocampal neurons. Insets show the initial rate of fluorescence increase $(p=0.64)$ and the time constant for endocytosis ( $p=0.54) . n=14$ coverslips per genotype The data represent mean \pm SEM. the possibility that the two mammalian isoforms might be redundant. We therefore generated double $\mathrm{KO}$ (DKO) mice lacking both isoforms, and performed field recordings in hippocampal slices. The input-output curve comparing the slope of the field potential to the strength of the fiber volley in LRRK1/2 DKO slices showed a significant main effect of genotype vs. WT (2-way ANOVA, $F_{(1,271)}=5.094$, $p=0.025)$, but post hoc tests showed no significant differences at any stimulation intensity (Figure $4 \mathbf{A}$ ). Consistent with the absence of change in baseline synaptic transmission, release probability assessed using the PPR shows no difference from WT in the LRRK1/2 DKO (Figure 4B). We also examined the LRRK1/2 DKO using cultured hippocampal neurons transfected with VGLUT1-pHluorin. Loss of LRRK1 does not by itself alter the initial rate of fluorescence increase or compensatory post-stimulus endocytosis (Figure 4C). We then examined the effect of LRRK1 inactivation in the LRRK2 KO background (LRRK1WT/LRRK2 KO vs. LRRK1 KO/LRRK2 KO). Loss of both isoforms also failed to alter either the fluorescence increase or decrease after stimulation (Figure 4D), demonstrating that LRRK1 does not compensate for the loss of LRRK2.

\section{Loss of LRRK2 Accelerates Endocytosis in Striatal Neurons}

Several studies have indicated high levels of native LRRK2 expression in the striatum (Melrose et al., 2006; Higashi et al., 2007; Westerlund et al., 2008; Giesert et al., 2013), which contains predominantly GABA neurons, suggesting that these cells may be more affected by the loss of LRRK2. Thus, we also examined the synaptic vesicle cycle in cultured striatal neurons from WT and LRRK2 $\mathrm{KO}$ mice, using the VGAT tagged with pHluorin at the lumenal/extracellular C-terminus (VGAT-pHluorin; Santos et al., 2013). The rate of both fluorescence increase and compensatory endocytosis after stimulation at $10 \mathrm{~Hz}$ for $60 \mathrm{~s}$ did not significantly change with loss of LRRK2, but endocytosis showed a trend to acceleration (Figure 5A). Previous studies using a shorter train of higher frequency stimulation $(20 \mathrm{~Hz}$ for $15 \mathrm{~s}$ ) have suggested that loss of LRRK2 inhibits endocytosis in rat striatal neurons (Arranz et al., 2015). Surprisingly, stimulation of mouse striatal neurons transfected with VGAT-pHluorin at $20 \mathrm{~Hz}$ for $15 \mathrm{~s}$ shows a significantly slower rate of fluorescence increase in LRRK2 KO than WT neurons, with a clear acceleration of compensatory endocytosis after the stimulus (Figure 5B). To determine whether loss of LRRK2 might also affect exocytosis in striatal neurons, we stimulated at $20 \mathrm{~Hz}$ in the presence of bafilomycin. Under these conditions, there was no difference in the rate or extent of exocytosis between WT and LRRK2 KO neurons (Figure 5C), supporting our conclusion that loss of LRRK2 does not directly affect the rate of exocytosis. Since stimulation at $20 \mathrm{~Hz}$ seemed to reveal a bigger difference in endocytosis between genotypes, we stimulated with sequential trains of 200 stimuli at 5-40 Hz (Figure 5D). As previously reported for hippocampal neurons, endocytosis was slower at higher stimulus frequencies in both genotypes $\left(F_{(3,88)}=6.753\right.$, 
A
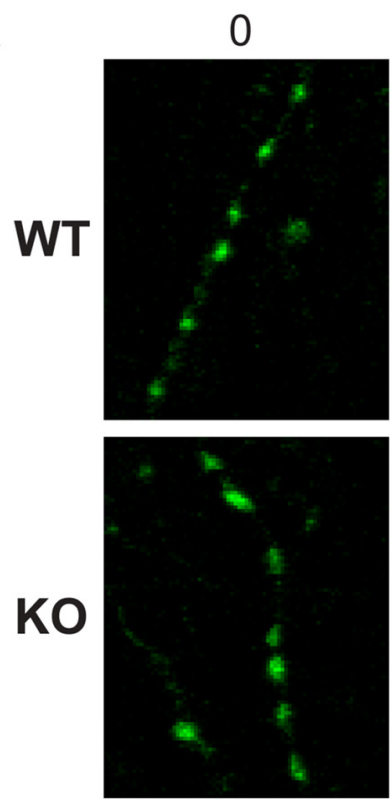

3.5
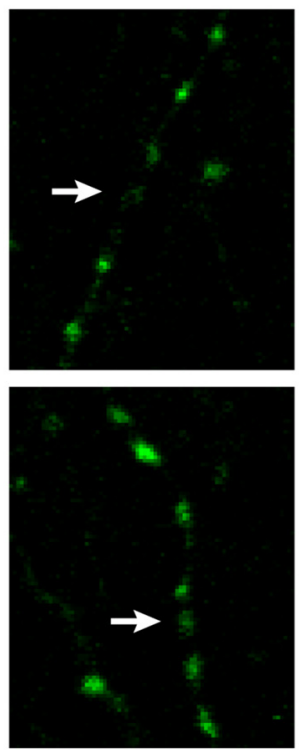

7.1
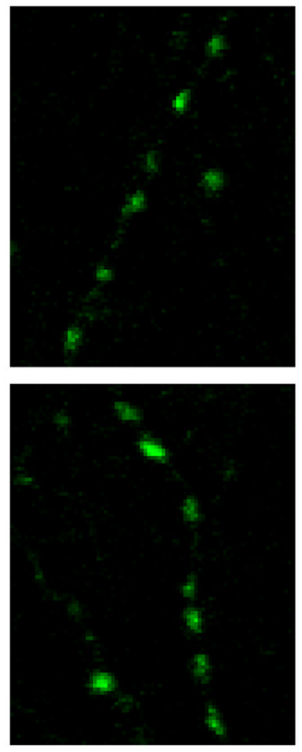

\section{$14.9 \mathrm{~s}$}
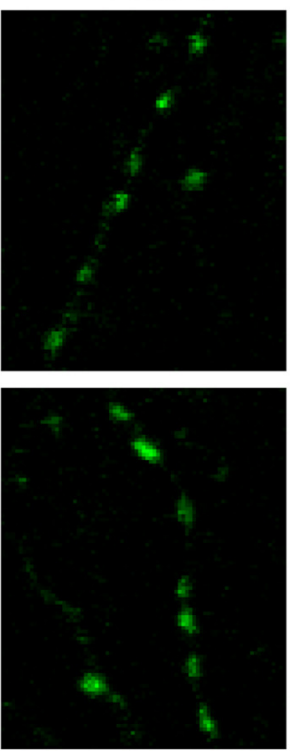

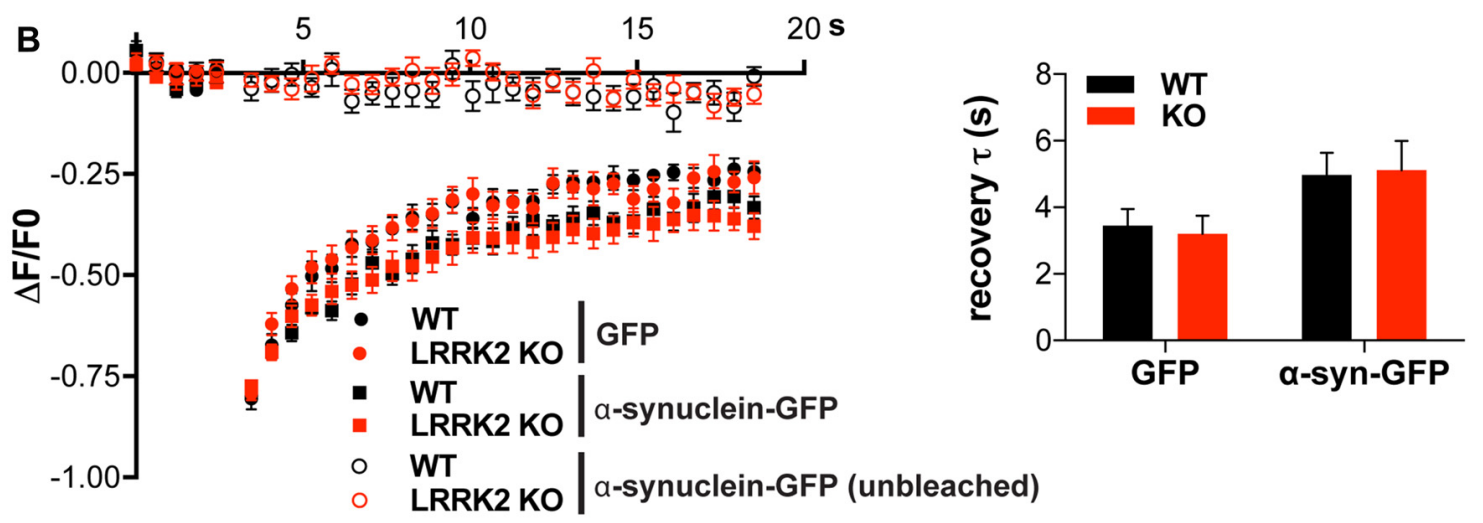

FIGURE 3 | Loss of LRRK2 does not alter the mobility of $\alpha$-synuclein ( $\alpha$-syn). (A) In either WT or LRRK2 KO hippocampal neurons, a single presynaptic bouton expressing $\alpha$-syn-GFP was photobleached (white arrow) and the recovery of fluorescence monitored at 400 ms intervals. (B) Hippocampal neurons from either WT or LRRK2 KO mice were transfected with GFP or $\alpha$-syn-GFP and the average change in fluorescence recorded before and after photobleaching (boutons bleached at $3.5 \mathrm{~s}$ after start of imaging). Nearby unbleached boutons expressing or $\alpha$-syn-GFP are shown as control. Inset shows the time constant for fluorescence recovery (WT vs. KO: $F_{(1,60)}=0.09, p=0.77$; GFP vs. $\alpha$-syn-GFP: $F_{(1,60)}=6.68, p=0.012 ; n=16$ boutons per genotype). The data represent mean \pm SEM.

$p<0.0004$; Armbruster et al., 2013; Kononenko et al., 2014; Figure 5D). However, LRRK2 genotype affected the rate of endocytosis across all stimulation frequencies $\left(F_{(1,88)}=08.703\right.$, $p=0.0041)$. Although fluorescence appears to decay below baseline after $5 \mathrm{~Hz}$ stimulation, the baseline is in fact elevated due to incomplete decay after an initial stimulus (data not shown): in striatal neurons, fluorescence often fails to recover fully to the pre-stimulus baseline after a single stimulus train (Figures 5A,B); with subsequent stimulation, the fluorescence decays to the original pre-stimulus baseline (Figure 5D). In contrast to striatal neurons, stimulation of hippocampal neurons transfected with VGLUT1-pHluorin at $20 \mathrm{~Hz}$ for $15 \mathrm{~s}$ showed no difference between genotype in either the rate of fluorescence increase or post-stimulus endocytosis (Figure 2D). Thus, the effect of LRRK2 on rate of endocytosis depends on neuron identity, not stimulation frequency.

\section{DISCUSSION}

The results indicate that LRRK2 has a modest inhibitory effect on endocytosis in striatal but not hippocampal neurons. In hippocampal slices, loss of LRRK2 has no consistent effect on basal synaptic transmission. We also failed to detect any change in synaptic vesicle exo- or endocytosis in hippocampal neurons from LRRK2 $\mathrm{KO}$ mice using both FM dye and more sensitive pHluorin-based reporters. The analysis of LRRK1/2 DKO mice 
A

B
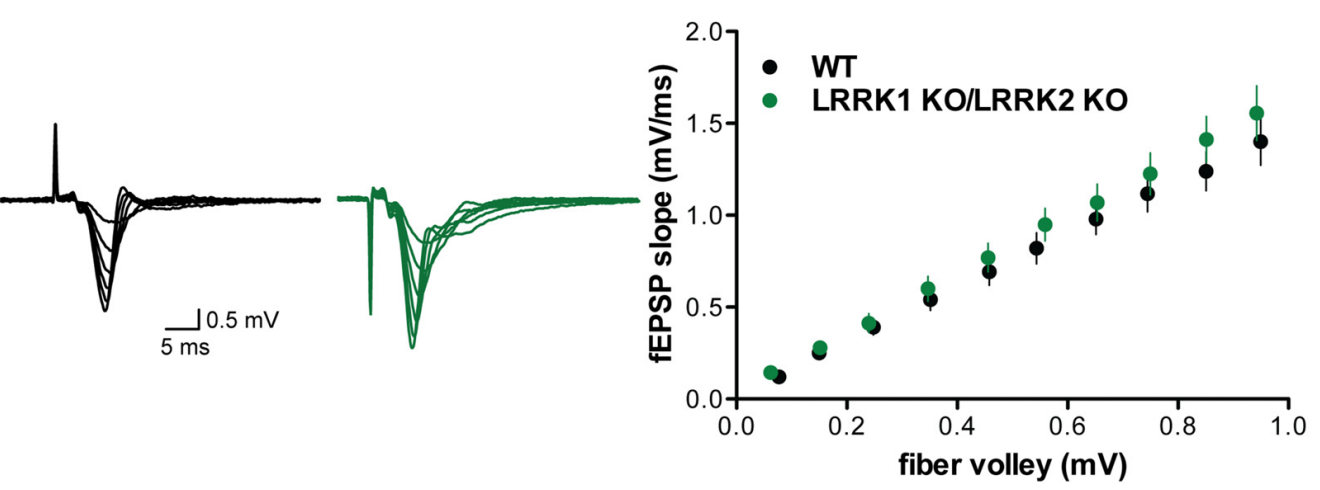

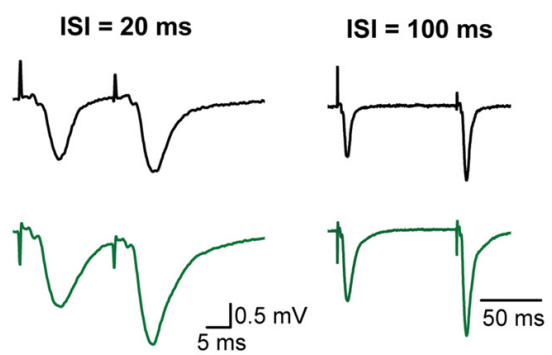

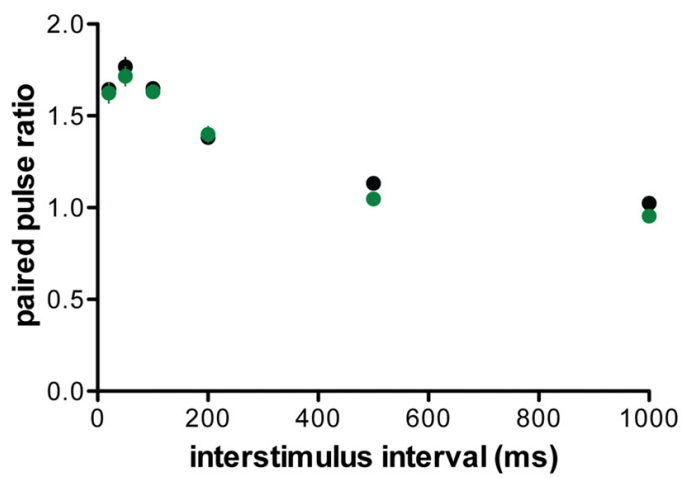

C
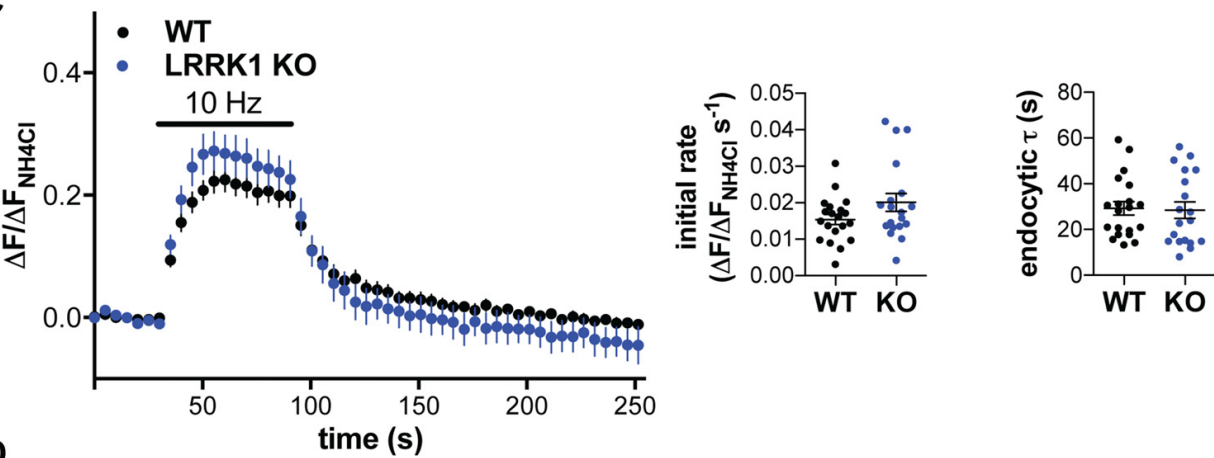

D
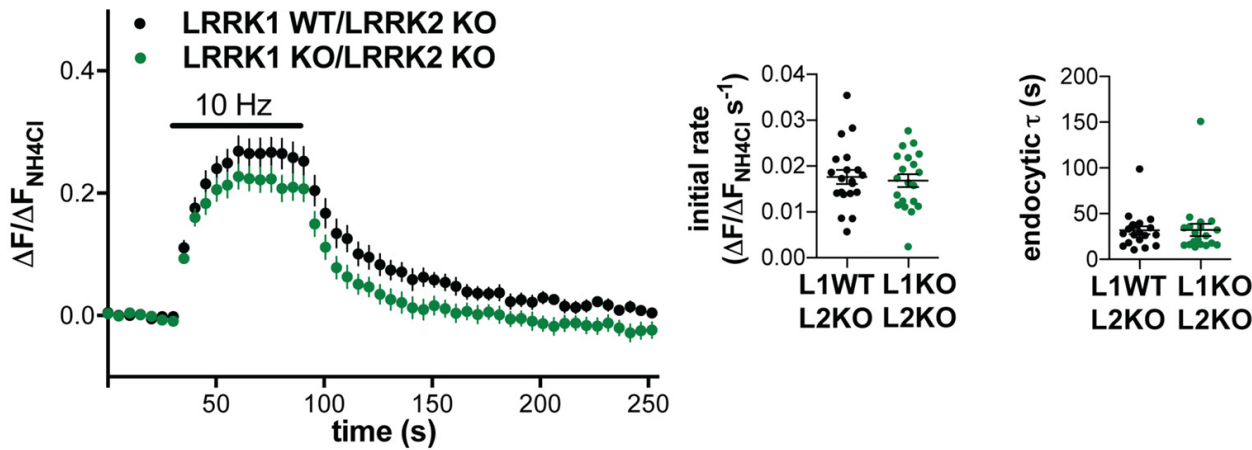

FIGURE 4 | Loss of LRRK1 and LRRK2 does not alter basal synaptic transmission or the synaptic vesicle cycle. (A) Representative traces (left) and input-output curve (right) of fEPSP slope normalized to fiber volley in hippocampal slices from WT (black) and LRRK1 KO/LRRK2 KO (green) mice $(F(1,271)=5.094$, $p=0.025 n=15$ slices from 2 to 3 animals per genotype). (B) Left, representative traces of field potentials from WT (black) and LRRK1 KO/LRRK2 KO (green) slices stimulated at ISI of 20 and $100 \mathrm{~ms}$. Right, paired pulse ratio does not differ between WT and LRRK1 KO/LRRK2 KO hippocampal slices $(F(1,168)=2.99, p=0.086$, $n=15$ slices from 2 to 3 animals per genotype). Fluorescence of VGLUT1-pHluorin in response to $10 \mathrm{~Hz}$ stimulation for $60 \mathrm{~s}$ in WT or LRRK1 KO (C) and LRRK1 WT/LRRK2 KO or LRRK1 KO/LRRK2 KO hippocampal neurons (D). Insets show the initial rate of fluorescence increase ( $p=0.09$ for $\mathbf{C}, 0.69$ for $\mathbf{D})$ and the time constant for endocytosis ( $p=0.86$ for $\mathbf{C}, 0.98$ for $\mathbf{D} ; n=19-20$ coverslips per genotype). The data represent mean \pm SEM. 

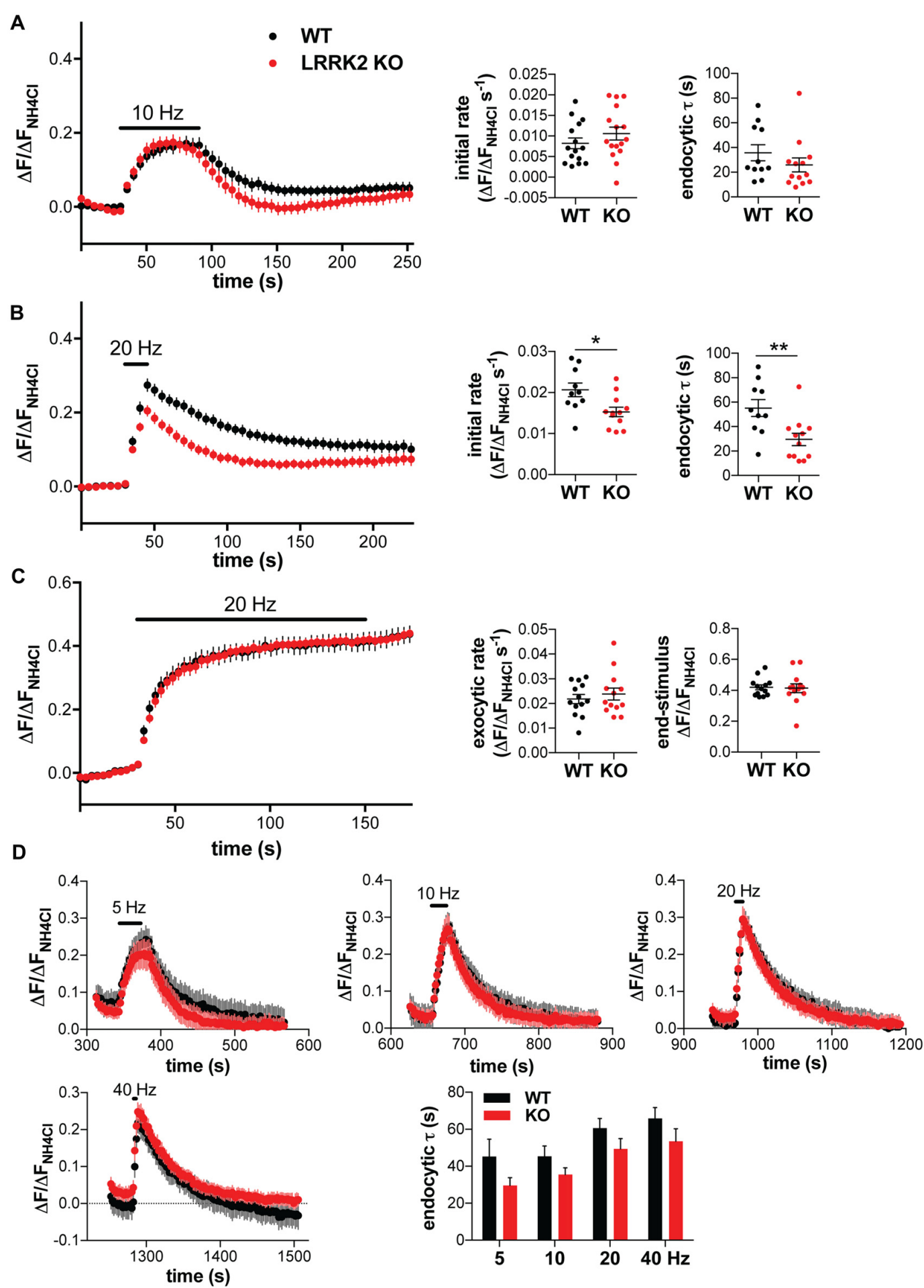

FIGURE 5 | Loss of LRRK2 accelerates synaptic vesicle endocytosis in striatal neurons. Response of vesicular GABA transporter (VGAT)-pHluorin to stimulation at $10 \mathrm{~Hz}$ for $60 \mathrm{~s}$ (A) or $20 \mathrm{~Hz}$ for $15 \mathrm{~s}$ (B) in transfected WT or LRRK2 KO striatal neurons. Insets show initial rate of fluorescence increase $(p=0.25$ for A, 0.014 for $\mathbf{B}$ ) and the time constant for endocytosis ( $D=0.26$ for $\mathbf{A}, 0.0064$ for $\mathbf{B}$ ). $n=15-16$ coverslips per genotype for (A), $10-12$ coverslips per genotype for (B); ${ }^{*} p<0.05$ by $t$-test, ${ }^{* *} p<0.01$ by $t$-test. (C) Response of VGAT-pHluorin to $20 \mathrm{~Hz}$ stimulation for $150 \mathrm{~s}$ in the presence of bafilomycin in transfected WT or LRRK2 KO striatal neurons. Insets show the initial exocytic rate $(p=0.52)$ and the fluorescence at the end of stimulation $(p=0.87)$. $n=13$ coverslips per genotype. (D) Response of VGAT-pHluorin to sequential trains of 200 stimuli at increasing frequency $(5 \mathrm{~Hz}$ for $40 \mathrm{~s}, 10 \mathrm{~Hz}$ for $20 \mathrm{~s}, 20 \mathrm{~Hz}$ for $10 \mathrm{~s}$ and $40 \mathrm{~Hz}$ for $5 \mathrm{~s})$ in transfected WT or LRRK2 KO striatal neurons. The time constant for endocytosis demonstrates a significant main effect of genotype $\left(F_{(1,88)}=08.703, p=0.0041\right)$ and stimulus frequency $\left(F_{(3,88)}=6.753, p<0.0004 ; n=11-13\right.$ coverslips per genotype). The data represent mean \pm SEM. 
shows that residual LRRK1 does not compensate for the loss of LRRK2. Further, LRRK2 does not affect the mobility of $\alpha$-syn and, by inference, its association with presynaptic membranes (Fortin et al., 2004; Unni et al., 2010). In striatal neurons, however, loss of LRRK2 leads to acceleration of endocytosis.

Why would we observe a bigger role for LRRK2 in striatal than hippocampal neurons? Striatal spiny projection neurons express LRRK2 at particularly high levels (Melrose et al., 2006; Higashi et al., 2007; Westerlund et al., 2008; Giesert et al., 2013). An effect on endocytosis is thus more likely to become apparent in striatal than hippocampal neurons, which express LRRK2 at lower levels. We cannot exclude the possibility that the difference reflects the use of different reporters (VGLUT1- vs. VGAT-pHluorin). However, previous work has shown that these two reporters have identical kinetics in hippocampal neurons (Santos et al., 2013). Thus, the differences we observe are unlikely to reflect the use of different reporters. Furthermore, experiments to assess the kinetics of VGAT-pHluorin specifically in GABAergic neurons of the hippocampus are technically challenging since they represent only $6 \%$ of the neurons in hippocampal cultures (Benson et al., 1994). The results thus support a larger role for LRRK2 in striatal neurons, which are largely GABAergic, than in hippocampal neurons, which are largely glutamatergic, presumably due to differential expression of LRRK2.

Studies to date on the role of LRRK2 in modulating presynaptic function have reported contradictory results. In the first studies suggesting a synaptic role for LRRK2, transgenic overexpression of WT LRRK2 enhanced striatal dopamine release, whereas overexpression of mutant LRRK2 had the opposite effect (Li et al., 2009, 2010). In the LRRK2 KO, evoked dopamine release was unaffected (Hinkle et al., 2012). However, changes in the amount of dopamine released could reflect alterations that do not involve the release machinery. Electrophysiologic recording from hippocampal slices of aged transgenic mice overexpressing G2019S LRRK2 has shown enhanced basal transmission, but without effect on paired pulse facilitation, suggesting a post- rather than presynaptic change (Sweet et al., 2015). Our study now provides the first analysis of the LRRK2 $\mathrm{KO}$ in hippocampal slices, with no clear effects on baseline excitatory transmission. In accord with these results, striatal slices from LRRK2 KO mice have shown no change in spontaneous release or paired pulse ratio (Parisiadou et al., 2014; Beccano-Kelly et al., 2015).

To image release directly, we and others have used cultured neurons. Using pHluorin-based reporters, overexpression of WT or mutant LRRK2 (G2019S) has been reported to slow endocytosis in cultured rat hippocampal neurons, without affecting exocytosis (Shin et al., 2008). In contrast, cortical neurons from transgenic mice overexpressing mutant LRRK2 (G2019S), have shown modest acceleration of endocytosis (Belluzzi et al., 2016). Although it is difficult to reconcile these results, over-expression of mutant LRRK2 also exerts toxicity (MacLeod et al., 2006; Smith et al., 2006). To study the function of LRRK2 without this complication, several groups have knocked down LRRK2 using siRNA, finding both increased paired-pulse depression (Piccoli et al., 2011) and reduced endocytosis without effects on exocytosis (Shin et al., 2008). Off-target effects of the RNAi may contribute to the apparent discrepancies. Consistent with the majority of these results, we find that LRRK2 does not modulate synaptic vesicle exocytosis. We cannot exclude specific effects on the readily releasable pool, but analysis of the recycling pool shows no changes that would suggest such a specific effect.

Avoiding the problems associated with overexpression and RNAi, analysis of Drosophila lacking the single LRRK gene has shown defects in endocytosis. LRRK apparently phosphorylates the BAR (bin-amphiphysin-rev) domain protein endophilin, which has a well established role in endocytosis (Matta et al., 2012). Consistent with the work in Drosophila, striatal neurons from LRRK2 KO rats also show slowed endocytosis (Arranz et al., 2015). In contrast, we observe accelerated endocytosis in the LRRK2 KO. Although we used the same striatal cultures and stimulation frequency, we imaged mouse rather than rat neurons, and included a small number of cortical neurons that provide excitatory drive and may make the networks more robust (Penrod et al., 2015). We also used VGAT-pHluorin rather than synaptophysin- and synaptobrevin-pHluorin (Shin et al., 2008; Arranz et al., 2015), although these reporters generally behave in very similar ways. In addition, we have varied a number of conditions, demonstrating that the observed increase in endocytosis occurs across multiple stimulation frequencies, but not in hippocampal neurons which express less LRRK2. It nonetheless remains possible that the role for both phosphorylation (by LRRK2) and dephosphorylation of endophilin proposed by Matta et al. (2012) could result in opposing effects of the KO under different circumstances.

Although the effect of LRRK2 on endocytosis in striatal neurons was only significant with $20 \mathrm{~Hz}$ stimulation, stimulation at $10 \mathrm{~Hz}$ also shows a trend to faster endocytosis. To investigate a frequency dependence of the effect, we also stimulated with 200 action potentials at $5-40 \mathrm{~Hz}$, and observed faster endocytosis in the $\mathrm{KO}$ at all frequencies. Further, stimulation of hippocampal neurons at $20 \mathrm{~Hz}$ showed no effect of the KO. We conclude that neuronal identity rather than stimulation frequency is critical to detect an effect of the $\mathrm{KO}$ on endocytosis. The work thus indicates a role for endogenous LRRK2 in the slowing of endocytosis. The toxicity asssociated with LRRK2 over-expression makes it difficult to draw conclusions about direct effects, but the phenotype of the $\mathrm{KO}$ predicts that a gain of LRRK2 function may also inhibit endocytosis. Consistent with this, loss-of-function mutations in the endocytic proteins synaptojanin and auxilin cause inherited forms of parkinsonism (Edvardson et al., 2012; Quadri et al., 2013). Thus, the inhibition of endocytosis by LRRK2 is consistent with other genetic mechanisms for this form of degeneration.

Although many neuronal populations including midbrain dopamine and hippocampal neurons express LRRK2, high levels of expression in the basal ganglia suggest a particularly important role for striatal LRRK2 in PD (Melrose et al., 2006; Higashi et al., 2007; Westerlund et al., 2008; Giesert et al., 2013). Direct pathway neurons in the striatum project to substantia nigra where they form synapses onto multiple neuronal populations including 
dopamine neurons (Watabe-Uchida et al., 2012; Calabresi et al., 2014), suggesting that presynaptic LRRK2 might influence striatal input to the midbrain. Alternatively, a disturbance in striatal LRRK2 might exert retrograde effects on presynaptic dopamine terminals in the striatum. Indeed, altered expression of LRRK2 in muscle acts in a retrograde fashion to influence transmitter release at the Drosophila neuromuscular junction (Penney et al., 2016), and mutations in LRRK2 lead to increased input from the cortex to striatum (Matikainen-Ankney et al., 2016). Thus, striatal LRRK2 may affect dopaminergic input through a similar, retrograde mechanism.

In conclusion, endogenous LRRK2 normally acts to slow endocytosis in GABAergic striatal neurons but not glutamatergic hippocampal neurons, consistent with the high levels of LRRK2 normally expressed in the striatum and with a non-cellautonomous role for LRRK2 mutations in the degeneration of dopamine neurons.

\section{REFERENCES}

Anwar, S., Peters, O., Millership, S., Ninkina, N., Doig, N., Connor-Robson, N., et al. (2011). Functional alterations to the nigrostriatal system in mice lacking all three members of the synuclein family. J. Neurosci. 31, 7264-7274. doi: 10.1523/JNEUROSCI.6194-10.2011

Armbruster, M., Messa, M., Ferguson, S. M., De Camilli, P., and Ryan, T. A. (2013). Dynamin phosphorylation controls optimization of endocytosis for brief action potential bursts. Elife 2:e00845. doi: 10.7554/eLife.00845

Arranz, A. M., Delbroek, L., Van Kolen, K., Guimarães, M. R., Mandemakers, W., Daneels, G., et al. (2015). LRRK2 functions in synaptic vesicle endocytosis through a kinase-dependent mechanism. J. Cell Sci. 128, 541-552. doi: $10.1242 /$ jcs. 158196

Beccano-Kelly, D. A., Volta, M., Munsie, L. N., Paschall, S. A., Tatarnikov, I., Co, K., et al. (2015). LRRK2 overexpression alters glutamatergic presynaptic plasticity, striatal dopamine tone, postsynaptic signal transduction, motor activity and memory. Hum. Mol. Genet. 24, 1336-1349. doi: $10.1093 / \mathrm{hmg} / \mathrm{ddu} 543$

Belluzzi, E., Gonnelli, A., Cirnaru, M. D., Marte, A., Plotegher, N., Russo, I., et al. (2016). LRRK2 phosphorylates pre-synaptic N-ethylmaleimide sensitive fusion (NSF) protein enhancing its ATPase activity and SNARE complex disassembling rate. Mol. Neurodegener. 11:1. doi: 10.1186/s13024-0150066-z

Benson, D. L., Watkins, F. H., Steward, O., and Banker, G. (1994). Characterization of GABAergic neurons in hippocampal cell cultures. J. Neurocytol. 23, 279-295. doi: $10.1007 /$ bf01188497

Biskup, S., Moore, D. J., Celsi, F., Higashi, S., West, A. B., Andrabi, S. A., et al. (2006). Localization of LRRK2 to membranous and vesicular structures in mammalian brain. Ann. Neurol. 60, 557-569. doi: 10.1002/ana.21019

Burré, J., Sharma, M., Tsetsenis, T., Buchman, V., Etherton, M., and Südhof, T. C. (2010). $\alpha$-synuclein promotes SNARE-complex assembly in vivo and in vitro. Science 329, 1663-1667. doi: 10.1126/science.1195227

Calabresi, P., Picconi, B., Tozzi, A., Ghiglieri, V., and Di Filippo, M. (2014). Direct and indirect pathways of basal ganglia: a critical reappraisal. Nat. Neurosci. 17, 1022-1030. doi: 10.1038/nn.3743

Cirnaru, M. D., Marte, A., Belluzzi, E., Russo, I., Gabrielli, M., Longo, F., et al. (2014). LRRK2 kinase activity regulates synaptic vesicle trafficking and neurotransmitter release through modulation of LRRK2 macromolecular complex. Front. Mol. Neurosci. 7:49. doi: 10.3389/fnmol.2014. 00049

Davidson, W. S., Jonas, A., Clayton, D. F., and George, J. M. (1998). Stabilization of $\alpha$-synuclein secondary structure upon binding to synthetic membranes. J. Biol. Chem. 273, 9443-9449. doi: 10.1074/jbc.273.16.9443

Dodson, M. W., Zhang, T., Jiang, C., Chen, S., and Guo, M. (2012). Roles of the Drosophila LRRK2 homolog in Rab7-dependent lysosomal positioning. Hum. Mol. Genet. 21, 1350-1363. doi: 10.1093/hmg/ddr573

\section{AUTHOR CONTRIBUTIONS}

JWM designed and conducted the imaging experiments, analyzed the data and wrote the manuscript. JY designed and conducted the slice electrophysiology experiments and analyzed the data. RHE oversaw the design of experiments and data analysis, and wrote the manuscript.

\section{FUNDING}

This work was supported by the following awards: Bumpus Foundation Postdoctoral Fellowship, Wheeler Center Fellowship and the McCamish Young Investigator Award from the National Parkinson Foundation to JWM; Grant no. R01 NS062715 from National Institutes of Health (NIH), a grant from the UCSF Weill Institute for Neurosciences and the John and Helen Cahill Family Endowment for Research on PD to RHE.

Edvardson, S., Cinnamon, Y., Ta-Shma, A., Shaag, A., Yim, Y. I., Zenvirt, S., et al. (2012). A deleterious mutation in DNAJC6 encoding the neuronalspecific clathrin-uncoating co-chaperone auxilin, is associated with juvenile parkinsonism. PLoS One 7:e36458. doi: 10.1371/journal.pone.0036458

Fernández-Alfonso, T., and Ryan, T. A. (2004). The kinetics of synaptic vesicle pool depletion at CNS synaptic terminals. Neuron 41, 943-953. doi: 10.1016/s0896-6273(04)00113-8

Fortin, D. L., Nemani, V. M., Voglmaier, S. M., Anthony, M. D., Ryan, T. A., and Edwards, R. H. (2005). Neural activity controls the synaptic accumulation of $\alpha$-synuclein. J. Neurosci. 25, 10913-10921. doi: 10.1523/JNEUROSCI.2922-05. 2005

Fortin, D. L., Troyer, M. D., Nakamura, K., Kubo, S., Anthony, M. D., and Edwards, R. H. (2004). Lipid rafts mediate the synaptic localization of $\alpha$-synuclein. J. Neurosci. 24, 6715-6723. doi: 10.1523/JNEUROSCI.1594-04. 2004

George, J. M., Jin, H., Woods, W. S., and Clayton, D. F. (1995). Characterization of a novel protein regulated during the critical period for song learning in the zebra finch. Neuron 15, 361-372. doi: 10.1016/0896-6273(95)90040-3

Giasson, B. I., Covy, J. P., Bonini, N. M., Hurtig, H. I., Farrer, M. J., Trojanowski, J. Q., et al. (2006). Biochemical and pathological characterization of Lrrk2. Ann. Neurol. 59, 315-322. doi: 10.1002/ana.20791

Giesert, F., Hofmann, A., Bürger, A., Zerle, J., Kloos, K., Hafen, U., et al. (2013). Expression analysis of Lrrk1, Lrrk2 and Lrrk2 splice variants in mice. PLoS One 8:e63778. doi: 10.1371/journal.pone.0063778

Gómez-Suaga, P., Rivero-Ríos, P., Fdez, E., Blanca Ramirez, M., Ferrer, I., Aiastui, A., et al. (2014). LRRK2 delays degradative receptor trafficking by impeding late endosomal budding through decreasing Rab7 activity. Hum. Mol. Genet. 23, 6779-6796. doi: 10.1093/hmg/ddu395

Greten-Harrison, B., Polydoro, M., Morimoto-Tomita, M., Diao, L., Williams, A. M., Nie, E. H., et al. (2010). $\alpha \beta \gamma$-Synuclein triple knockout mice reveal age-dependent neuronal dysfunction. Proc. Natl. Acad. Sci. U S A 107, 19573-19578. doi: 10.1073/pnas.1005005107

Healy, D. G., Falchi, M., O'Sullivan, S. S., Bonifati, V., Durr, A., Bressman, S., et al. (2008). Phenotype, genotype and worldwide genetic penetrance of LRRK2associated Parkinson's disease: a case-control study. Lancet Neurol. 7, 583-590. doi: 10.1016/S1474-4422(08)70117-0

Higashi, S., Moore, D. J., Colebrooke, R. E., Biskup, S., Dawson, V. L., Arai, H., et al. (2007). Expression and localization of Parkinson's disease-associated leucine-rich repeat kinase 2 in the mouse brain. J. Neurochem. 100, 368-381. doi: 10.1111/j.1471-4159.2006.04246.x

Hinkle, K. M., Yue, M., Behrouz, B., Dächsel, J. C., Lincoln, S. J., Bowles, E. E., et al. (2012). LRRK2 knockout mice have an intact dopaminergic system but display alterations in exploratory and motor co-ordination behaviors. Mol. Neurodegener. 7:25. doi: 10.1186/1750-1326-7-25

Iwai, A., Masliah, E., Yoshimoto, M., Ge, N., Flanagan, L., de Silva, H. A., et al. (1995). The precursor protein of non-A $\beta$ component of Alzheimer's disease 
amyloid is a presynaptic protein of the central nervous system. Neuron 14, 467-475. doi: 10.1016/0896-6273(95)90302-X

Janezic, S., Threlfell, S., Dodson, P. D., Dowie, M. J., Taylor, T. N., Potgieter, D., et al. (2013). Deficits in dopaminergic transmission precede neuron loss and dysfunction in a new Parkinson model. Proc. Natl. Acad. Sci. U S A 110, E4016-E4025. doi: 10.1073/pnas.1309143110

Kononenko, N. L., Puchkov, D., Classen, G. A., Walter, A. M., Pechstein, A., Sawade, L., et al. (2014). Clathrin/AP-2 mediate synaptic vesicle reformation from endosome-like vacuoles but are not essential for membrane retrieval at central synapses. Neuron 82, 981-988. doi: 10.1016/j.neuron.2014.05.007

Lee, H. J., Choi, C., and Lee, S. J. (2002). Membrane-bound $\alpha$-synuclein has a high aggregation propensity and the ability to seed the aggregation of the cytosolic form. J. Biol. Chem. 277, 671-678. doi: 10.1074/jbc.M107045200

Li, Y., Liu, W., Oo, T. F., Wang, L., Tang, Y., Jackson-Lewis, V., et al. (2009). Mutant LRRK2(R1441G) BAC transgenic mice recapitulate cardinal features of Parkinson's disease. Nat. Neurosci. 12, 826-828. doi: 10.1038/nn.2349

Li, X., Patel, J. C., Wang, J., Avshalumov, M. V., Nicholson, C., Buxbaum, J. D., et al. (2010). Enhanced striatal dopamine transmission and motor performance with LRRK2 overexpression in mice is eliminated by familial Parkinson's disease mutation G2019S. J. Neurosci. 30, 1788-1797. doi: 10.1523/JNEUROSCI.5604-09.2010

Lin, X., Parisiadou, L., Gu, X. L., Wang, L., Shim, H., Sun, L., et al. (2009). Leucinerich repeat kinase 2 regulates the progression of neuropathology induced by Parkinson's-disease-related mutant $\alpha$-synuclein. Neuron 64, 807-827. doi: 10.1016/j.neuron.2009.11.006

MacLeod, D., Dowman, J., Hammond, R., Leete, T., Inoue, K., and Abeliovich, A. (2006). The familial Parkinsonism gene LRRK2 regulates neurite process morphology. Neuron 52, 587-593. doi: 10.1016/j.neuron.2006.10.008

Martin, I., Kim, J. W., Lee, B. D., Kang, H. C., Xu, J. C., Jia, H., et al. (2014). Ribosomal protein $\mathrm{s} 15$ phosphorylation mediates LRRK2 neurodegeneration in Parkinson's disease. Cell 157, 472-485. doi: 10.1016/j.cell.2014.01.064

Matikainen-Ankney, B. A., Kezunovic, N., Mesias, R. E., Tian, Y., Williams, F. M., Huntley, G. W., et al. (2016). Altered development of synapse structure and function in striatum caused by Parkinson's disease-linked LRRK2-G2019S mutation. J. Neurosci. 36, 7128-7141. doi: 10.1523/JNEUROSCI.3314-15.2016

Matta, S., Van Kolen, K., da Cunha, R., van den Bogaart, G., Mandemakers, W., Miskiewicz, K., et al. (2012). LRRK2 controls an EndoA phosphorylation cycle in synaptic endocytosis. Neuron 75, 1008-1021. doi: 10.1016/j.neuron.2012. 08.022

Melrose, H., Lincoln, S., Tyndall, G., Dickson, D., and Farrer, M. (2006). Anatomical localization of leucine-rich repeat kinase 2 in mouse brain. Neuroscience 139, 791-794. doi: 10.1016/j.neuroscience.2006.01.017

Miesenböck, G., De Angelis, D. A., and Rothman, J. E. (1998). Visualizing secretion and synaptic transmission with $\mathrm{pH}$-sensitive green fluorescent proteins. Nature 394, 192-195. doi: 10.1038/28190

Nakata, Y., Yasuda, T., Fukaya, M., Yamamori, S., Itakura, M., Nihira, T., et al. (2012). Accumulation of $\alpha$-synuclein triggered by presynaptic dysfunction. J. Neurosci. 32, 17186-17196. doi: 10.1523/JNEUROSCI.2220-12.2012

Nemani, V. M., Lu, W., Berge, V., Nakamura, K., Onoa, B., Lee, M. K., et al. (2010). Increased expression of $\alpha$-synuclein reduces neurotransmitter release by inhibiting synaptic vesicle reclustering after endocytosis. Neuron $65,66-79$. doi: 10.1016/j.neuron.2009.12.023

Orenstein, S. J., Kuo, S. H., Tasset, I., Arias, E., Koga, H., Fernandez-Carasa, I., et al. (2013). Interplay of LRRK2 with chaperone-mediated autophagy. Nat. Neurosci. 16, 394-406. doi: 10.1038/nn.3350

Parisiadou, L., Yu, J., Sgobio, C., Xie, C., Liu, G., Sun, L., et al. (2014). LRRK2 regulates synaptogenesis and dopamine receptor activation through modulation of PKA activity. Nat. Neurosci. 17, 367-376. doi: 10.1038/nn.3636

Penney, J., Tsurudome, K., Liao, E. H., Kauwe, G., Gray, L., Yanagiya, A., et al. (2016). LRRK2 regulates retrograde synaptic compensation at the Drosophila neuromuscular junction. Nat. Commun. 7:12188. doi: 10.1038/ncomms 12188

Penrod, R. D., Campagna, J., Panneck, T., Preese, L., and Lanier, L. M. (2015). The presence of cortical neurons in striatal-cortical co-cultures alters the effects of dopamine and BDNF on medium spiny neuron dendritic development. Front. Cell. Neurosci. 9:269. doi: 10.3389/fncel.2015.00269

Piccoli, G., Condliffe, S. B., Bauer, M., Giesert, F., Boldt, K., De Astis, S., et al. (2011). LRRK2 controls synaptic vesicle storage and mobilization within the recycling pool. J. Neurosci. 31, 2225-2237. doi: 10.1523/JNEUROSCI.3730-10. 2011

Piccoli, G., Onofri, F., Cirnaru, M. D., Kaiser, C. J., Jagtap, P., Kastenmüller, A., et al. (2014). Leucine-rich repeat kinase 2 binds to neuronal vesicles through protein interactions mediated by its C-terminal WD40 domain. Mol. Cell. Biol. 34, 2147-2161. doi: 10.1128/MCB.00914-13

Polymeropoulos, M. H., Lavedan, C., Leroy, E., Ide, S. E., Dehejia, A., Dutra, A., et al. (1997). Mutation in the $\alpha$-synuclein gene identified in families with Parkinson's disease. Science 276, 2045-2047. doi: 10.1126/science.276.5321. 2045

Quadri, M., Fang, M., Picillo, M., Olgiati, S., Breedveld, G. J., Graafland, J., et al. (2013). Mutation in the SYNJ1 gene associated with autosomal recessive, earlyonset Parkinsonism. Hum. Mutat. 34, 1208-1215. doi: 10.1002/humu.22373

Santos, M. S., Park, C. K., Foss, S. M., Li, H., and Voglmaier, S. M. (2013). Sorting of the vesicular GABA transporter to functional vesicle pools by an atypical dileucine-like motif. J. Neurosci. 33, 10634-10646. doi: 10.1523/JNEUROSCI. 0329-13.2013

Scott, D. A., Tabarean, I., Tang, Y., Cartier, A., Masliah, E., and Roy, S. (2010). A pathologic cascade leading to synaptic dysfunction in $\alpha$-synuclein-induced neurodegeneration. J. Neurosci. 30, 8083-8095. doi: 10.1523/JNEUROSCI. 1091-10.2010

Shin, N., Jeong, H., Kwon, J., Heo, H. Y., Kwon, J. J., Yun, H. J., et al. (2008). LRRK2 regulates synaptic vesicle endocytosis. Exp. Cell Res. 314, 2055-2065. doi: 10.1016/j.yexcr.2008.02.015

Singleton, A. B., Farrer, M., Johnson, J., Singleton, A., Hague, S., Kachergus, J., et al. (2003). $\alpha$-Synuclein locus triplication causes Parkinson's disease. Science 302:841. doi: 10.1126/science.1090278

Smith, W. W., Pei, Z., Jiang, H., Dawson, V. L., Dawson, T. M., and Ross, C. A. (2006). Kinase activity of mutant LRRK2 mediates neuronal toxicity. Nat. Neurosci. 9, 1231-1233. doi: 10.1038/nn1776

Spillantini, M. G., Crowther, R. A., Jakes, R., Hasegawa, M., and Goedert, M. (1998). $\alpha$-Synuclein in filamentous inclusions of Lewy bodies from Parkinson's disease and dementia with lewy bodies. Proc. Natl. Acad. Sci. U S A 95, 6469-6473. doi: 10.1073/pnas.95.11.6469

Spillantini, M. G., Schmidt, M. L., Lee, V. M., Trojanowski, J. Q., Jakes, R., and Goedert, M. (1997). $\alpha$-synuclein in Lewy bodies. Nature 388, 839-840. doi: $10.1038 / 42166$

Sweet, E. S., Saunier-Rebori, B., Yue, Z., and Blitzer, R. D. (2015). The Parkinson's disease-associated mutation LRRK2-G2019S impairs synaptic plasticity in mouse hippocampus. J. Neurosci. 35, 11190-11195. doi: 10.1523/JNEUROSCI. 0040-15.2015

Unni, V. K., Weissman, T. A., Rockenstein, E., Masliah, E., McLean, P. J., and Hyman, B. T. (2010). In vivo imaging of $\alpha$-synuclein in mouse cortex demonstrates stable expression and differential subcellular compartment mobility. PLoS One 5:e10589. doi: 10.1371/journal.pone.0010589

Voglmaier, S. M., Kam, K., Yang, H., Fortin, D. L., Hua, Z., Nicoll, R. A., et al. (2006). Distinct endocytic pathways control the rate and extent of synaptic vesicle protein recycling. Neuron 51, 71-84. doi: 10.1016/j.neuron.2006.05.027

Watabe-Uchida, M., Zhu, L., Ogawa, S. K., Vamanrao, A., and Uchida, N. (2012). Whole-brain mapping of direct inputs to midbrain dopamine neurons. Neuron 74, 858-873. doi: 10.1016/j.neuron.2012.03.017

Westerlund, M., Belin, A. C., Anvret, A., Bickford, P., Olson, L., and Galter, D. (2008). Developmental regulation of leucine-rich repeat kinase 1 and 2 expression in the brain and other rodent and human organs: implications for Parkinson's disease. Neuroscience 152, 429-436. doi: 10.1016/j.neuroscience. 2007.10.062

Conflict of Interest Statement: The authors declare that the research was conducted in the absence of any commercial or financial relationships that could be construed as a potential conflict of interest.

Copyright (c) 2017 Maas, Yang and Edwards. This is an open-access article distributed under the terms of the Creative Commons Attribution License (CC BY). The use, distribution and reproduction in other forums is permitted, provided the original author(s) or licensor are credited and that the original publication in this journal is cited, in accordance with accepted academic practice. No use, distribution or reproduction is permitted which does not comply with these terms. 